\title{
Adoption of Artificial Intelligence for Diagnosis and Treatment of Staphylococcus aureus Infections Disease on Humans
}

\author{
Kanayo Kizito Uka1 ${ }^{*}$, Stanley Ikechukwu Oguoma1, Chekwube Alphonsus Chukwu2, \\ Chijioke Izuchukwu Emele ${ }^{1}$ \\ ${ }^{1}$ Department of Computer Science, Imo State University, Owerri, Nigeria \\ ${ }^{2}$ Nnamdi Azikiwe Teaching Hospital, Nnewi, Nigeria \\ Email: ^kizzyict@gmail.com, oguomaikechukwu@gmail.com,dr.alphons@yahoo.com, chijiokeemele@gmail.com
}

How to cite this paper: Uka, K.K., Oguoma, S.I., Chukwu, C.A. and Emele, C.I. (2020) Adoption of Artificial Intelligence for Diagnosis and Treatment of Staphylococcus aureus Infections Disease on $\mathrm{Hu}-$ mans. E-Health Telecommunication Systems and Networks, 9, 1-15.

https://doi.org/10.4236/etsn.2020.91001

Received: February 16, 2020

Accepted: March 27, 2020

Published: March 30, 2020

Copyright $\odot 2020$ by author(s) and Scientific Research Publishing Inc. This work is licensed under the Creative Commons Attribution International License (CC BY 4.0).

http://creativecommons.org/licenses/by/4.0/

\begin{abstract}
The aim of this paper is to develop an expert system that could aid medical practitioner to effectively diagnose and treat Staphylococcus aureus infections disease on a human. The objective of the research includes to develop an expert system for quick diagnosis and detection of Staphylococcus aureus bacteria on human skin, a system that aids in accurate treatment of staph infectious diseases by doctors, helps in quick decision making in the hospital, improves accuracy in drug prescription, and a system that will bring about computerized storage process, and to enlighten the knowledge workers on how to implement a computer based decision support systems and importance of it in the health care. The research was motivated due to delay in diagnosis and identification of Staphylococcus aureus bacteria and the fast rate at which infectious disease is spreading, delay in treatment of these bacteria, increase of guess work by health practitioners leading to delay in decision making and lack of electronic storage facility in the hospitals. Top down approach was used in the system design of this research while adopting expert system as the methodology and the programming language used was Java and database design used was MySQL. The result after design was a computerized standalone application that assists health practitioners (Doctor's) in quick identification, diagnosis, prescription and treatment of Staphylococcus aureus bacteria on human skin. The expert system will facilitate quick decision making in the clinic.
\end{abstract}

\section{Keywords}

Artificial Intelligence, Expert System, Diagnosis, Treatment, Staphylococcus aureus, Infectious Disease 


\section{Introduction}

Staphylococcus aureus as stated by Wikipedia [1] is a gram-positive, round-shaped bacterium that is an associate of the Firmicutes. Furthermore, it is also an associate-member of the normal vegetation of the body mainly found in human nose, respiratory tract, and the skin. It can also be positive for catalase or nitrate reduction and also a facultative anaerobe that could grow without the need for oxygen [2]. Staphylococcus aureus is part of the normal human flora (bacteria that normally reside in or on humans) and does not usually cause infection. It can be said that bacteria living in any human body without affecting or causing infection, is known as "colonization" [3]. Made us to understand that humans are most often colonized with $S$. aureus in their noses, hence these bacteria can also be found on the human skin and other body sites. According to the Minnesota Department of health report on Staphylococcus aureus infectious disease in 2019 , it stated that over $20 \%$ of their population almost always be colonized with $S$. aureus, while $60 \%$ of the population will be colonized with $S$. aureus off and on, and another $20 \%$ are almost never colonized with $S$. aureus [3]. Doctors or a medical practitioner have always diagnosed patients of Staph either by conducting a culture from the area of suspected infection or through patient symptoms and the health care provider's evaluation based on their observation. The introduction of information communication technology has made it easier for most organizations/sector to carry out their jobs easily and accurately. Therefore, Staphylococcus aureus as bacteria found on human would be diagnosed with the help of an Artificial Intelligence so as to enable the medical practitioners take accurate evaluation and decision on the best method and drugs for the treatment of these bacteria. In this paper, a computer based diagnosis and treatment is presented in Figure 12 and Figure 13 with means of identifying the actual bacteria through the picture which will be shown once user enters a symptom. The diagnosis provides causes/symptom and best procedure to apply the antibiotic (drugs) for the treatment of the bacteria. In summary, the new expert system contributed a lot in the aspect of quick decision making by providing an interface for diagnosis and treatment of staph infectious disease. As we know, the world is rapidly changing because of the introduction of Artificial Intelligence (AI) in various aspect of life and hence, the health sector is not left out. The new system which was designed as an expert system enables medical doctors to carry out diagnosis easily, where patients are subjected to some questions and answers provided are used by the new system inference engine to proffer possible cause with image, and treatment that is appropriate for the disease. After a successful diagnosis by the doctor, a decision can now be taken by following the procedures given by the expert machine in treating the patient and hence reduce the time in carrying out laboratory test. The system also provides an interface for update in case any new diagnosis or treatment of the staph disease is identified. The expert system is standalone software designed with JAVA programming language and secured with a username and password combination. Once an access is success- 
ful, the user of the system can add new staph symptoms, causes of the illness and treatment of the staphylococcus infectious disease adequately. These paper is organized in sections such as: Introduction, Statement of Problem, Aim and Objectives of Study, Related Literature, methodology, Proposed system architecture, analysis of the existing system, analysis and operation of the new system, sequence diagram of the new system Diagnosis, sequence diagram of the new system Treatment, High level diagram of the new system, New system diagnosis and Treatment algorithm, Result, Conclusion and Recommendations For Further Studies.

\subsection{Statement of Problem}

The problem of the existing system as observed includes:

1) Delay in diagnosis and identification of Staphylococcus aureus bacteria on humans

2) Delay in treatment of these bacteria

3) High increment of guess work by most health practitioners

4) Wrong prescription of treatment of these infections

5) Poor method of keeping records

\subsection{Aim and Objectives of Study}

This paper is aimed at developing an expert system that could aid medical practitioner to effectively diagnose and treat Staphylococcus aureus infections on humans.

\section{The Objectives Are}

1) To develop an expert system for quick diagnosis and detection of Staphylococcus aureus bacteria on humans.

2) To provide a system that aid in accurate treatment of staph infectious diseases by doctors.

3) Provide a system that could facilitate decision making in the hospital administration in regards to infectious diseases.

4) To design a system that could improve accuracy in drug prescription and administration.

\section{Related Literature}

\subsection{Artificial Intelligence and Expert Systems}

[4] noted that intelligence is the ability for an object to learn and understand, also further to be able to solve problems and make decisions based on the understanding already gotten. In the other hand an Artificial Intelligence (AI) could be said to be an intelligence by a machine and to provide a mechanism for machines to do or solve things that would require intelligence if operated by humans. [4] also outlined a central problem of AI as being (locomotion), that is to say that $\mathrm{AI}$ includes traits such as reasoning, knowledge, planning, learning, communication, perception and the ability to move and manipulate objects. 
Looking at the early 90's, AI attained its highest feast in practical application, notwithstanding all its previous hindrances. It was effectively applied in logistics data mining and medical diagnosis; though AI success started being revitalized with the commercial attainment of expert systems. Expert system is a form of Artificial Intelligence program that stimulates the knowledge and analytical skills of one or more human experts.

Though [5] stated that conventional expert system is produced for a very narrow domain with obviously defined expertise whereby making the entire system performance fully reliant on the right choice of experts although a common strategy is to find just one expert, when a more complex expert system is being built or when expertise is not well defined, multiple experts might be needed.

\subsection{Types of Staphylococcus aureus Infections}

The infectious disease known as Staphylococcus aureus ranges from mild stage to life threatening issues and hence most common is the skin infections which are often caused by abscesses. Furthermore, the deadly disease could travel through the bloodstream to infect any part of the body, more particularly the heart valves which could also be referred to as the endocarditis and the bones (osteomyelitis). It is certain that staphylococcal infections react more probable in sure situations such as [6].

1) Bloodstream infections: This happens when a catheter inserted in the vein stayed there for a long time.

2) Endocarditis: This happens when illegal drugs are injected to people or when an artificial valve is used or when a used catheter in the vein is infected

3) Osteomyelitis: This type of infection happens when the Staphylococcus aureus enters into the bone, whereby forming infection in the bloodstream or causing an infection in adjacent soft tissue, these infections are found with people with deep pressure sores or foot sores due to cause of diabetes.

4) Lung Infection (Pneumonia): This is when people have had virus or bloodstream infection. It could also be referred to as when people start taking corticosteroids or drugs that suppress the human immune system.

Table 1 below shows other related literatures from other researchers on the diagnosis and treatment of Staphylococcus aureus infection disease on wound species. It highlighted the authors, title, year of publication and adopted method for treatment.

\subsection{Key Difference between the New System and the Related Studies}

1) Some of the related studies used a particular antibiotic for staph treatment such as $\beta$-cyclodextrin, Gentamicin, Norfloxacin, Amoxiclav, apoferritin-silver (I) ions and treated with a redo aortic valve replacement, patch false aneurysm and replacement ascending aorta. But the new system provides a platform for acquisition of knowledge in respect to the diagnosis and treatment of Staphylococcus aureus infectious disease. It stores procedures, teraphy, possible drugs 
Table 1. Further related literatures.

\begin{tabular}{|c|c|c|c|}
\hline Authors & Topic & Year & Method Used \\
\hline $\begin{array}{l}\text { Brook, } \\
\text { Vladimir, } \\
\text { and Juliane } \\
{[7]}\end{array}$ & $\begin{array}{l}\text { Prevention and Treatmen } \\
\text { of Staphylococcus aureus } \\
\text { Pneumonia With a } \\
\beta \text {-Cyclodextrin Derivative }\end{array}$ & 2010 & $\begin{array}{l}\beta \text {-cyclodextrin derivative IB201. The IB201 protects } \\
\text { against } S \text {. aureus pneumonia. (A) IB201 was } \\
\text { administered to mice via retro-orbital injection } 2 \mathrm{~h} \\
\text { after infection, } 12 \mathrm{~h} \text { after infection, and then every } 12 \\
\mathrm{~h} \text { thereafter. They were challenged with } S \text {. aureus } \\
\text { Newman via the i.n. route, and mortality was } \\
\text { recorded at } 24,48 \text {, and } 72 \text { hours postinfection } \\
\text { (P } 0.039 ; 15 \text { animals per group) }\end{array}$ \\
\hline $\begin{array}{l}\text { Annabel, } \\
\text { Edward, } \\
\text { Graham [8] }\end{array}$ & $\begin{array}{l}\text { Large false aneurysm } \\
\text { following aortic valve } \\
\text { replacement and excision } \\
\text { of an abscess cavity for } \\
\text { the treatment of } \\
\text { staphylococcus aureus } \\
\text { endocarditis }\end{array}$ & 2009 & $\begin{array}{l}\text { Successfully treated with a redo aortic valve } \\
\text { replacement, patch false aneurysm and replacement } \\
\text { ascending aorta }\end{array}$ \\
\hline $\begin{array}{l}\text { Dana et al., } \\
\text { [9] }\end{array}$ & $\begin{array}{l}\text { Electrochemical } \\
\text { Behaviour of Apoferritin } \\
\text { Encapsulating of Silver (I) } \\
\text { Ions and Its Application } \\
\text { for Treatment of } \\
\text { Staphylococcus aureus }\end{array}$ & 2012 & $\begin{array}{l}\text { Treatment of } S \text {. aureus with apoferritin-silver (I) ions } \\
\text { complex. (A) Growth curves of } S \text {. aureus with the } \\
\text { addition of apoferritin (AP) and silver (I) ions (Ag) in } \\
\text { the active concentration of } 7.5,15,30 \text { and } 60 \mu \mathrm{M} \text {. (B) } \\
\text { Changes in the dynamics of } S \text {. aureus growth after } 2.5 \\
\text { hour cultivation in the presence of silver (I) ions with } \\
\text { apoferritin. Growth curve of bacterial culture without } \\
\text { the silver (I) ions addition has been subtracted. }\end{array}$ \\
\hline $\begin{array}{l}\text { Ahmed et } \\
\text { al., [10] }\end{array}$ & $\begin{array}{l}\text { Incidence and Antibiotic } \\
\text { Susceptibility Profile of } \\
\text { Staphylococcus aureus } \\
\text { Isolates from Wounds } \\
\text { of Patients at Specialist } \\
\text { Hospital, Sokoto, Nigeria }\end{array}$ & 2017 & $\begin{array}{l}\text { All wound swabs obtained from patients with wound } \\
\text { infections during the study period were cultured on } \\
\text { mannitol salt agar media. The isolates were } \\
\text { identified using standard microbiological methods. } \\
\text { Antibiotic susceptibility test was carried out on the } \\
\text { identified isolates using the modified Kirby-Bauer } \\
\text { disc diffusion method and methicillin resistant } \\
\text { Staphylococcus aureus (MRSA) test was carried out } \\
\text { using Oxacillin agar screen test as described by } \\
\text { Clinical and Laboratory Standard Institute (CLSI, } \\
\text { 2016). Treated with Gentamicin, } \\
\text { Norfloxacin and Amoxiclav }\end{array}$ \\
\hline
\end{tabular}

(antibiotics) used in treating the disease. It is based on this system that quick treatment of the disease in accomplished faster.

2) Most of the related studies worked on remote systems, and not a standalone system which made it possible for outside cyber-attack. The attack was because of the online presences which allowed intruders to frequently manipulate private information.

3) It is also identified that related studies were not able to provide image of the type of staph disease for better understanding after diagnosis. It is much easier to come to a conclusion on an issue if a clearer picture of the stated problem, which is where the existing studies could not solve. Therefore the new system stated problems and solution accordingly with the pictorial view of the type of disease. 
4) The new system is a standalone application where only the doctor user can use the system which made it much more secured and hence facilitate quick decision making in the clinic.

\section{Methodology}

Artificial Intelligence (AI) has many branches such as: Neural Network, expert system, data mining, statistical Artificial Intelligence (AI), Pattern Recognition, Fuzzy Logic, Swarm Intelligent, and Genetic Algorithm. But this work will be adopting one of the branches of Artificial Intelligence called Expert system which is defined as an interactive and user friendly interface computer-based decision support system which uses facts and heuristics to solve difficult decision making problems. Figure 1 below illustrates the major components of an expert system. It is based on the components that the proposed diagnosis and treatment of staphylococcus aureus on human wound species was designed. As shown in Figure 1 below, the human expert in this case is the medical doctor who provides all the facts on how staphylococcus aureus infection on patients are diagnosed and treated, then the knowledge engineer acquire the information's (facts) concerning the patient through the knowledge acquisition process by either interviewing the human expert or asking questions, then all facts about the disease are store in the Knowledge Base (Database). The inference engine set the rules, which is the brain of the entire expert system operation, that is to say if the inference engine is faulty then the solution to diagnosis and treatment of the staph infection will be wrong. In this case, the inference engine provides the answer base on users request from the user interface by the end user. The process is done repeatedly by the end user of the system.

\subsection{Proposed System Architecture}

Figure 2 illustrates the proposed system architecture. The architecture contains all the modules in the proposed system. Once a user launch the application either as a doctor user or the system operator, the user must have a login combination of username and password. The sequence diagram in Figure 4 illustrates the full operation of the proposed system.

\subsection{Analysis of the Existing System}

Diagnosis and treatment of infectious disease like Staphylococcus aureus in most hospital are done with some laboratory equipment's. Activities like conducting a test with colonies, coagulase, hemolysins and thermostable deoxyribonuclease are regularly used to detect Staphylococcus aureus. But with the introduction of computer and Artificial Intelligence, most medical diagnosis and treatment are now simple, instead of undergoing a rigorous process just to identify a particular cure or treatment for a certain disease, expert system machines could help in facilitating the process by using already defined facts and rules used in handling such problems before to draw conclusion on what to do next time such issue is 


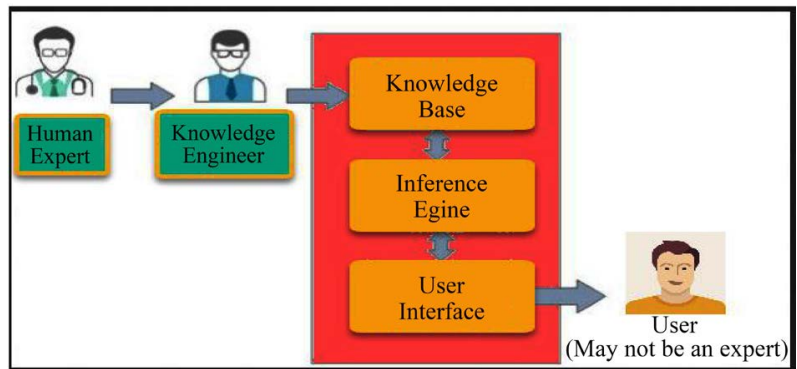

Figure 1. Overview of an expert system component (Source: [11]).

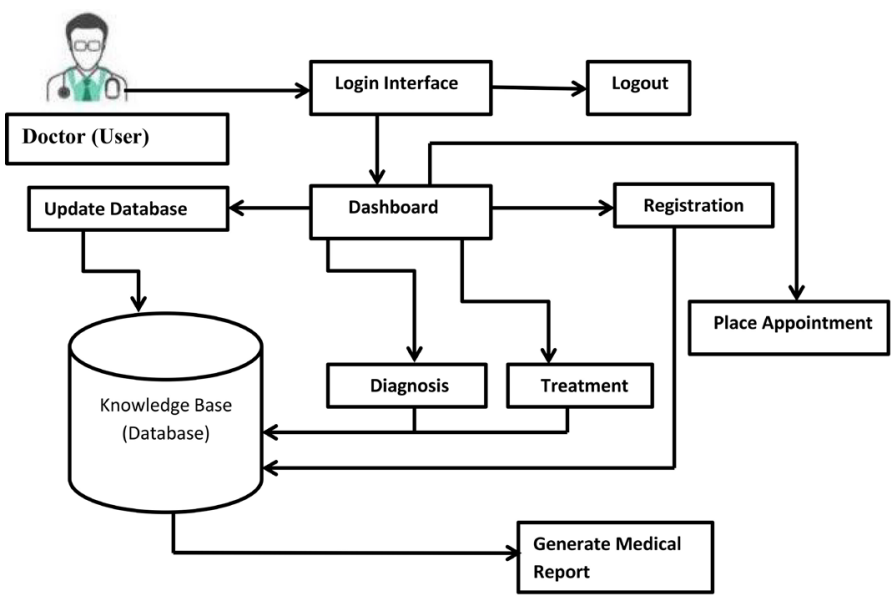

Figure 2. Proposed system architecture.

witnessed. The microbiologist enters into the laboratory to perform the same exact process often and often to diagnose or treat a staph patient. The doctor or lab scientist manually performs some physical diagnosis or examination on the patient by checking the skin lesion before proceeding to collection of some sample for testing. They check for tissue sample or nasal secretions for signs of the bacteria. After the result of the laboratory test is out, prescription of drugs (Treatment) commence which most times takes much time than expected.

\subsection{Analysis and Operation of the New System}

The new system is an Artificial Intelligence for Diagnosis and Treatment of Staphylococcus aureus on Human body. It has a friendly user interface designed with an object oriented high level programming language (JAVA). This language was used because of its ability to accurately model objects and its behaviours while the Knowledge base (Database) was done using My Structural Query Language (MySQL). Once a user launch the application from the desktop computer, the splash screen interface will be displayed after which a login interface will be shown. The login interface will authenticate user's information before access to the dashboard could be allowed. It is on the dashboard interface that the major operations of the expert system are done such as: updating the knowledge base (database) of the system. It is on the update database interface that the doctor as a user could add or remove facts like Staphylococcus aureus diagnosis and 
treatment procedure based on already existing diagnosis and treatment methods used in the hospital for treating staph patients. The registration interface helps to collect patients information (Health Record), Place appointment interface enable the user to book appointment for staph patient (if need arise), generate medical report is an interface used for getting medical history of patients while the diagnosis interfaces is used for carrying out diagnosis and treatment of patients. The diagnosis interface provides a friendly and interactive surface for question and answer by the doctor and patients. It is through this method that the doctor uses to collect or examine the patient and hence collects data. The treatment interface enables the user to proffer treatment and procedure to take in administering the drugs. After a successful process the user logout through the logout button.

\subsection{Sequence Diagram of the New System}

The sequence diagram of the new system is shown in Figure 3 and Figure 4 respectively; it shows an arrangement of object interactions between objects and its classes involved in the design of the new system. The sequence diagram explain how the doctor (user) could access the expert system application right from their personal device, how diagnosis and treatment of Staphylococcus aureus infection disease could be effectively treated. Other activities includes a sequence of how patients registration, placement of appointment and generation of medical report are done by the end user.

\subsubsection{Sequence Diagram of the New System Diagnosis}

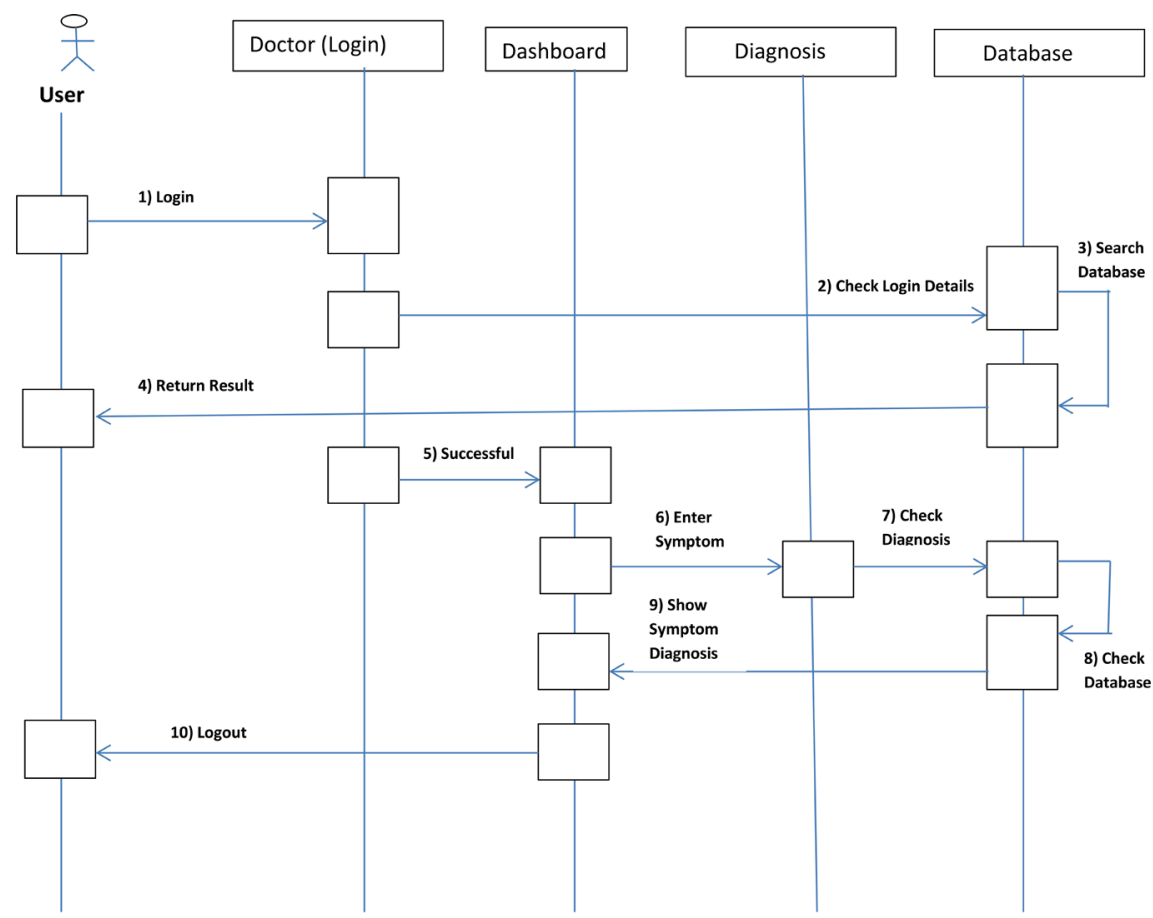

Figure 3. Sequence diagram of the new system diagnosis. 
3.4.2. Sequence Diagram of the New System Treatment

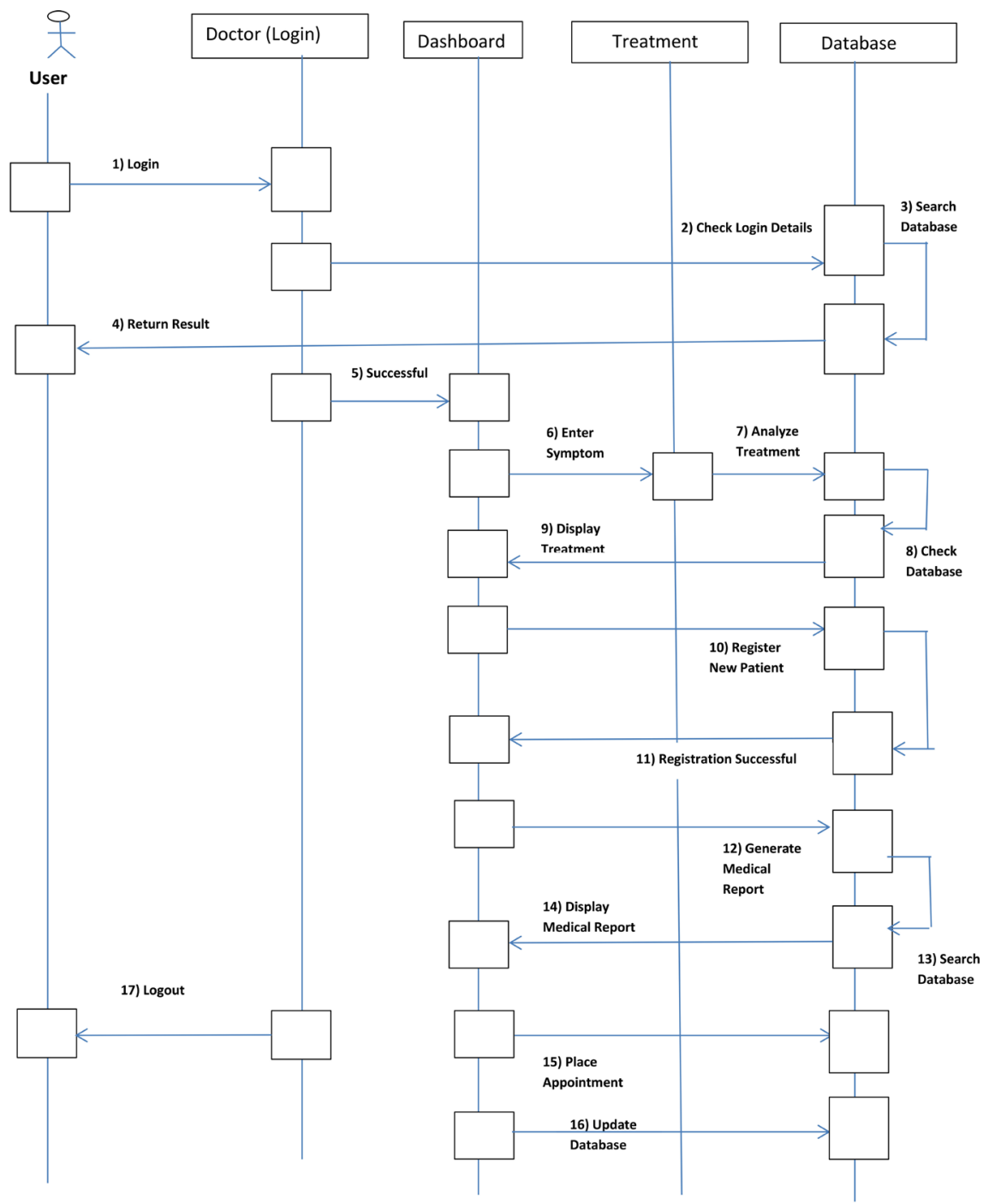

Figure 4. Sequence diagram of the proposed system treatment.

\subsection{High Level Model of the New System}

The proposed system high level model was designed following the top-down design approach, where every module, sub-module or sub-sub-module of the system is shown. Once a user successfully login into the dashboard which in this case link every other operation of the system as shown in Figure 4 and Figure 5 respectively, the diagnosis and treatment of any staph patient could be successfully achieved without further delay.

\subsection{New System Diagnosis and Treatment Algorithm}

Step 1. Start program;

2. Enter Symptom;

3. Process input;

4. if (symptom = "Boils on the hair follicle or oil gland") \{ 


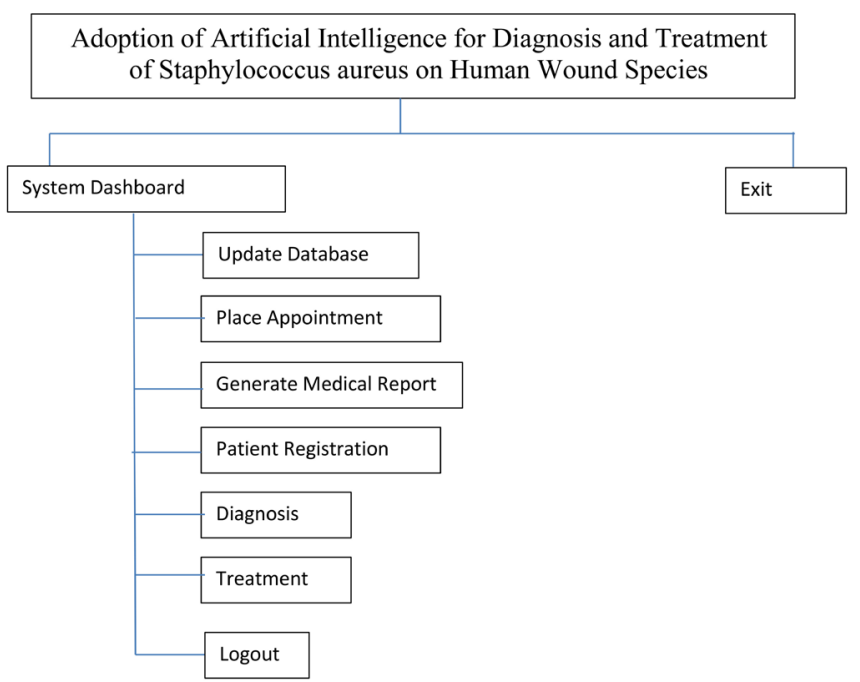

Figure 5. High level model of the proposed system.

Diagnosis = "A pocket of pus that develops in a hair follicle or oil gland. The skin over the infected area usually becomes red and swollen. If a boil breaks open, it will probably drain pus. Boils occur most often under the arms or around the groin or buttocks";

Diagnosis Image Sample $=$

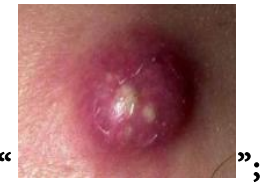

Treatment = "Intake of Vancomycin antibiotics increasingly";

\} if (symptom = "Painful Rash") \{

Diagnosis = "Impetigo: Painful Rash and large blisters that may ooze fluid and develop a honey-colored crust";

Diagnosis Image Sample $=$ “

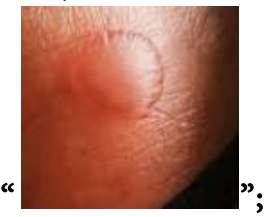

Treatment $=$ "Vancocin (vancomycin)";

\}

if (symptom = "Skin Redness and Swelling") \{

Diagnosis = "Cellulitis, it causes skin redness and swelling on the surface of your skin. Sores (ulcers) or areas of oozing discharge may develop. It is an infection of the deeper layers of skin";

Diagnosis Image Sample $=$ “

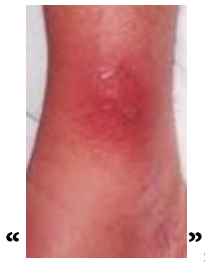

Treatment $=$ "Combination of Bactroban (mupirocin) and Nafcillin antibiotics"; 


\section{\} if (symptom = "Fever, Rash, Blisters")\{}

Diagnosis "Toxins produced as a result of a staph infection may lead to staphylococcal scalded skin syndrome. Affecting mostly babies and children, this condition features fever, a rash and sometimes blisters. When the blisters break, the top layer of skin comes off leaving a red, raw surface that looks like a burn";

Diagnosis Image Sample = " $>$ in;

Treatment = "Antibiotic ointment";

\} if (symptom = "Nausea and vomiting, Diarrhea, Dehydration, Low blood pressure")\{

Diagnosis "Food poisoning. Staph bacteria are one of the most common causes of food poisoning. Symptoms come on quickly, usually within hours of eating a contaminated food. Symptoms usually disappear quickly, too, often lasting just half a day";

Diagnosis Image Sample $=$ “

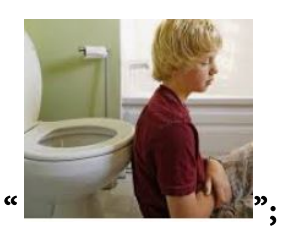

Treatment $=$ "Take food safety precautions. Such as: Wash your hands before handling food. If food will be out for a while, make sure that hot foods stay hot - above $140 \mathrm{~F}$ (60 C) - and that cold foods stay at $40 \mathrm{~F}$ (4.4 C) or below. Refrigerate leftovers as soon as possible";

\} else \{

Display: "No treatment yet";

end program;

Stop;

\section{Results}

The result of any system shows what the new system looks like after implementation in real life scenario. As stated earlier, the new system is designed with an object oriented programming language known as JAVA which produced a standalone application. After a successive installation, a quick launch will be initiated immediately which will trigger the first module, the Splash Screen Module shown in Figure 6. Figure 6 below will initiate the next module shown in Figure 7 which is the Application Login Interface where users of the system could provide username and password combination to login into the application dashboard. The dashboard shown in Figure 8, serves as the control center of the whole intelligent application for diagnosis and treatment of Staphylococcus aureus infectious disease. Figure 9 shows the new system Patient Registration interface. It is through this interface that patient medical histories are acquired. 
Figure 10 shows the designed interface of the new application patient medical report module, this interface enable user of the system to quickly generate medical report of any patient with his/her name. Figure 11 shows the new system database update interface. This interface provides the user access to delete facts, add new symptoms, treatments, problem of the ailment. Figure 12 shows the diagnosis of staph disease, this interface helps the doctor (User) to ask patients some questions and hence get response from them before proceeding to the next module for treatment of the disease. Once patients diagnosis is done, the treatment comes immediately shown in Figure 13 of this paper. The treatment interface displays symptom of the disease with a related image and procedure on the drugs to take for the cure. Then there might be need for the patient to meet with the doctor for medical checkup, hence the new system provides a module where placement of medical appointment could be recorded shown in Figure 14.

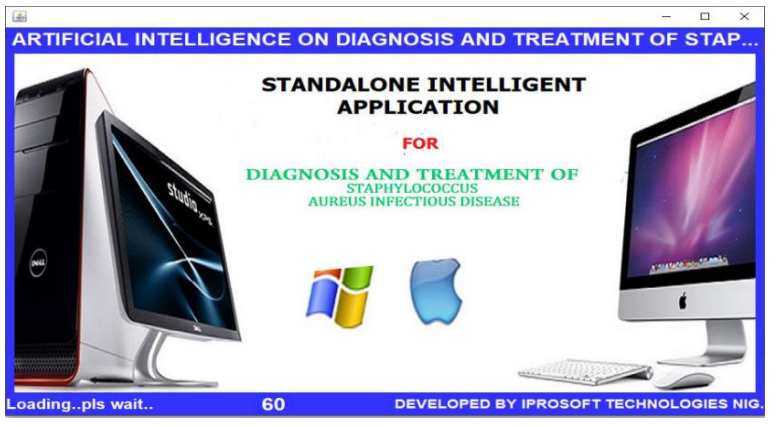

Figure 6. Splash Screen of the new System interface.

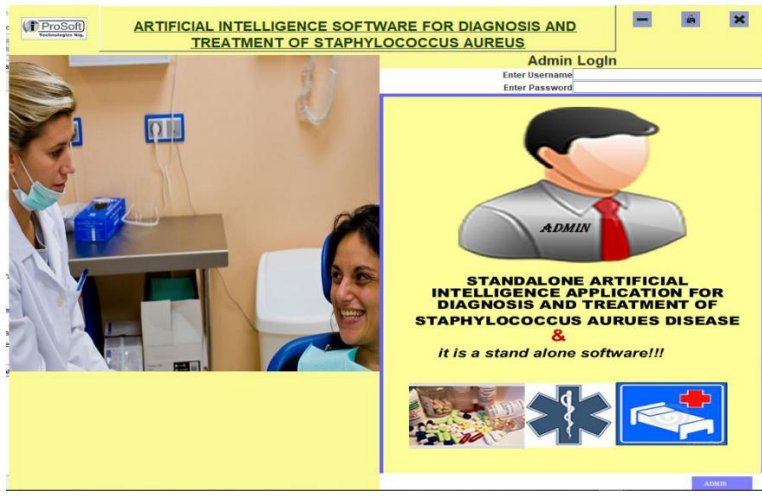

Figure 7. User application login interface.

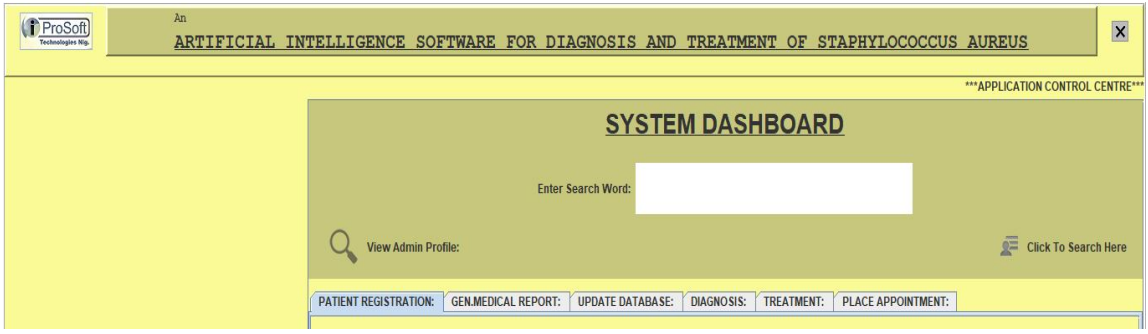

Figure 8. New system application dashboard interface. 


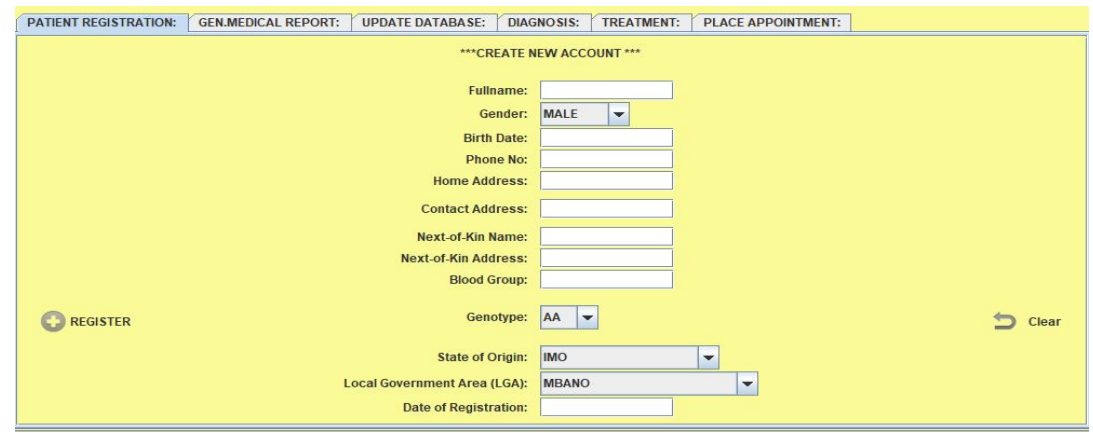

Figure 9. The new system patient registration interface.

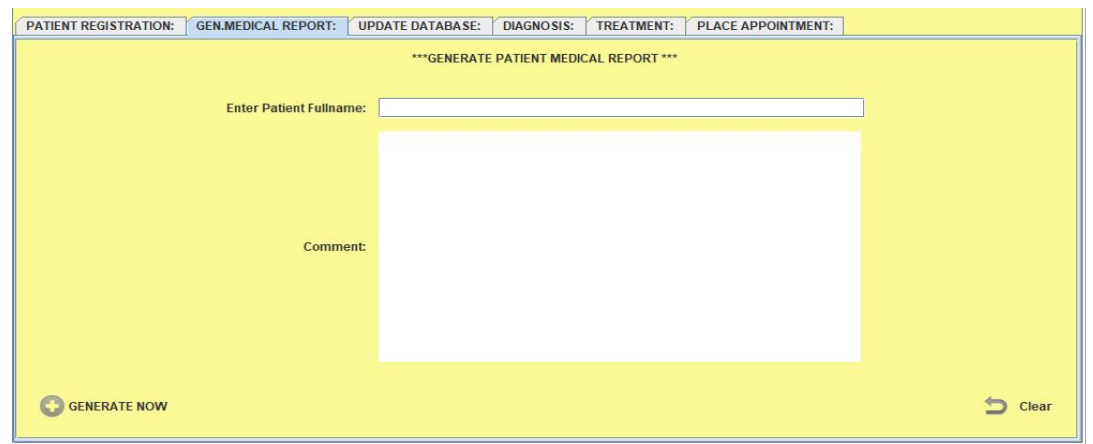

Figure 10. New system patient medical report interface.

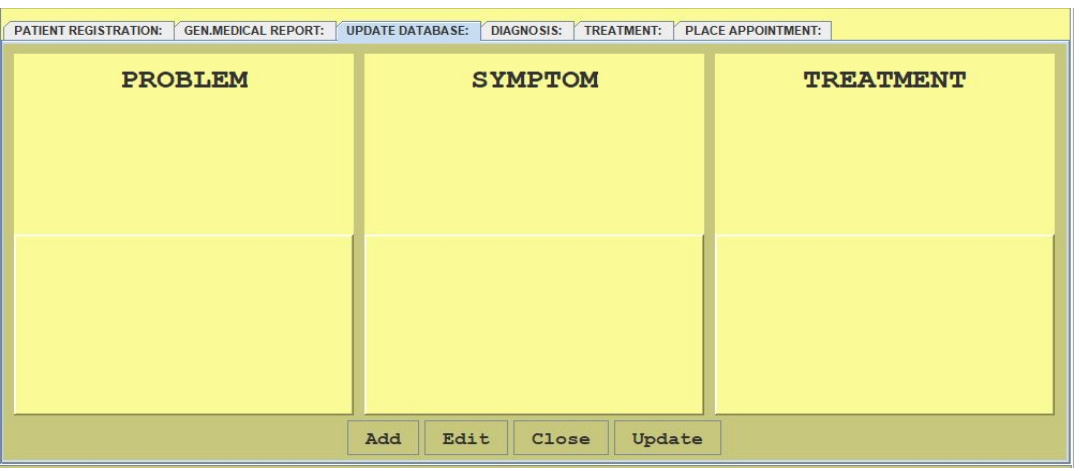

Figure 11. New system database update interface.

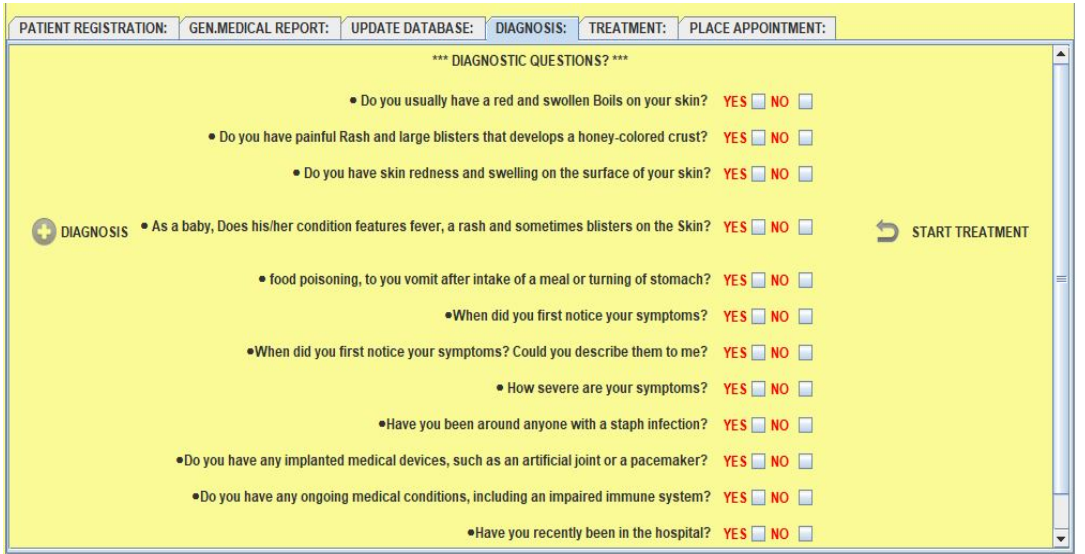

Figure 12. New system diagnosis interface. 


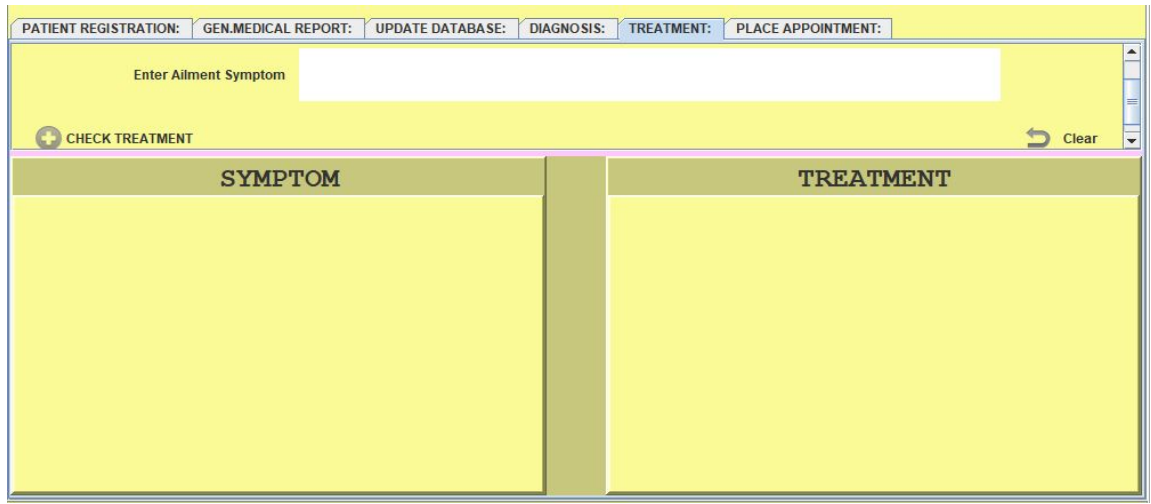

Figure 13. New system treatment interface.

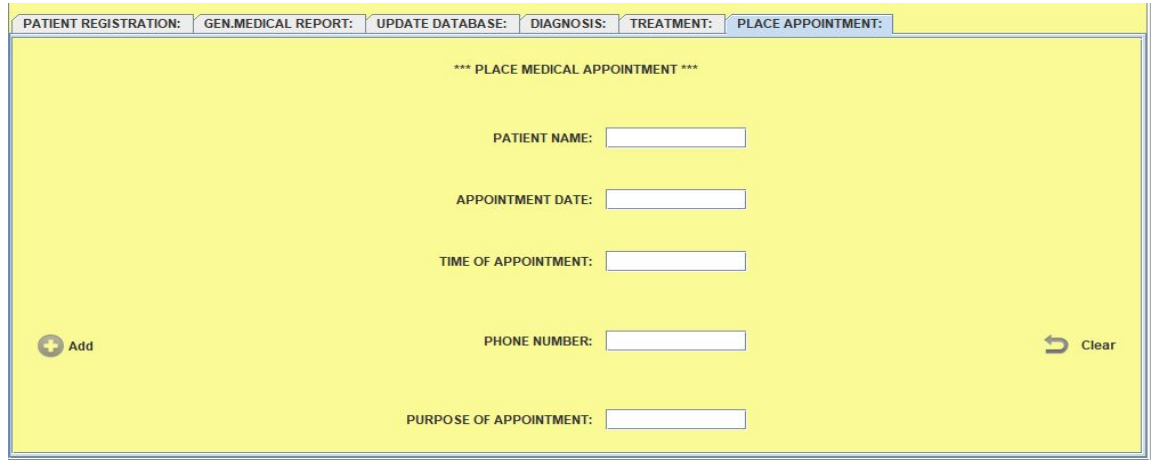

Figure 14. New system patient appointment placement interface.

\section{Conclusion}

Advancement of Artificial Intelligence in health sector and its significance cannot be over emphasized, and on the same hand application of AI in treating deadly infectious diseases like Staphylococcus aureus and its likes requires a quick response so as to provide instance treatment of the disease instead of wasting further time in laboratory experiments. The adoption of expert system which happens to be a branch of Artificial Intelligence enables the researcher to acquire accurate information on the diagnosis and treatment procedures in handling staph infectious disease clinically, where the knowledge is extracted and stored in the knowledge base (Database). Hence, the new system was able to carry out diagnosis and hence provide drugs (treatment) based on the result of the diagnosis. The diagnosis enables the patients to ask and answer some questions related to symptoms witnessed. After an interactive and friendly response, the system provides type of staph disease with an image of the staph disease.

\section{Recommendations for Further Studies}

Recommendations for further studies are as follows:

1) It will be a great study if further research be conducted in the aspect of making the work cloud base.

2) And more collaborative and AI supported device that could aid in diagnosis and treatment of infectious disease on patients. 


\section{Conflicts of Interest}

The authors declare no conflicts of interest regarding the publication of this paper.

\section{References}

[1] Wikipedia (2018) What Is Staphylococcus aureus. https://en.wikipedia.org/w/index.php?title=Staphylococcus_aureus\&oldid=8615969 9

[2] Masalha, M., Borovok, I., Schreiber, R., Aharonowitz, Y. and Cohen, G. (2001) Analysis of Transcription of the Staphylococcus aureus Aerobic Class Ib and Anaerobic Class III Ribonucleotide Reductase Genes in Response to Oxygen. Journal of Bacteriology, 183, 7260-7272. https://doi.org/10.1128/JB.183.24.7260-7272.2001 https://www.ncbi.nlm.nih.gov/pmc/articles/PMC95576

[3] Minnesota Department of Health (2019) Diagnosis and Treatment of Staphylococcus aureus. https://www.health.state.mn.us/diseases/staph/mrsa/index.html

[4] Alatishe, A.A., Adegbola, M.A. and Ike, D.U. (2013) File Sharing Application for Android. International Journal of Computer Science Engineering, 2, 20.

[5] Rajendran, R.K. and Rubenstein, D. (2004) Optimizing the Quality of Scalable Video Streams on P2P Networks. Proceedings of GLOBECOM, Vol. 2, 953-959. https://doi.org/10.1145/1005686.1005735

[6] Bush, L.M. (2018) Staphylococcus aureus Infections. https://www.msdmanuals.com/home/infections/bacterial-infections-gram-positivebacteria/staphylococcus-aureus-infections

[7] Ragle, B.E., Karginov, V.A. and Wardenburg, J.B. (2010) Prevention and Treatment of Staphylococcus aureus Pneumonia with a $\beta$-Cyclodextrin Derivative. Antimicrobial Agents and Chemotherapy, 54, 298-304. https://doi.org/10.1128/AAC.00973-09

[8] Sharkey, A.J., Peng, E.W.K. and Cooper, G.J. (2009) Large False Aneurysm Following Aortic Valve Replacement and Excision of an Abscess Cavity for the Treatment of Staphylococcus aureus Endocarditis. European Journal of Cardio-Thoracic Surgery, 35, 1079. https://doi.org/10.1016/j.ejcts.2009.01.054

[9] Dospivova, D., Hynek, D., Kopel, P., Bezdekova, A., Sochor, J., Krizkova, S., Adam, V., Trnkova, L., Hubalek, J., Babula, P., Provaznik, I., Vrba, R. and Kizek, R. (2012) Electrochemical Behaviour of Apoferritin Encapsulating of Silver(I) Ions and Its Application for Treatment of Staphylococcus aureus. International Journal of Electrochemical Science, 7, 6378-6395. http://www.electrochemsci.org

[10] Olowo-okere, A., Atata, R.F., Suleiman, S. and Ungokore, H.Y. (2017) Incidence and Antibiotic Susceptibility Profile of Staphylococcus aureus Isolates from Wounds of Patients at Specialist Hospital, Sokoto, Nigeria. Journal of Medical Bacteriology, 6, 44-50. https://www.researchgate.net/publication/321864136

[11] Guru99, Component of Expert System. https://www.guru99.com/expert-systems-with-applications.html 